\title{
Anticancer effect of quinacrine on diffuse large B-cell lymphoma via inhibition of MSI2-NUMB signaling pathway
}

\author{
SHUJUN YANG, LIXIA SHENG, KAIHONG XU, YI WANG, HUILING ZHU, \\ PING ZHANG, QITIAN MU and GUIFANG OUYANG
}

Department of Hematology, Ningbo First Hospital, Ningbo, Zhejiang 315010, P.R. China

Received May 17, 2017; Accepted September 8, 2017

DOI: $10.3892 / \mathrm{mmr} .2017 .7892$

\begin{abstract}
Diffuse large B-cell lymphoma (DLBCL) is the most common subtype of non-Hodgkin's lymphoma. Despite improvements in the clinical outcomes of DLBCL, 30\% of patients will develop relapse/refractory disease. Therefore, novel therapeutic drugs have been investigated to improve disease outcomes. Previous studies have revealed the anticancer effects of quinacrine (QC) on tumor cells in vitro, although its role in human DLBCL is yet to be identified. The present study sought to examine the cytotoxic effect of QC on DLBCL cells. QC induced G0/G1 cell cycle arrest and apoptosis in the DLBCL cell lines SU-DHL-8 and OCI-LY01, in a dose-dependent manner, in addition to the downregulation of cyclin-dependent kinase 4/6 and the upregulation of cleaved poly-ADP ribose polymerase 1. Upon exposure to QC, RNA-binding protein Musashi homolog 2 inactivation and activation of protein numb homolog were observed. In addition, QC was able to inhibit the expression of Myc proto-oncogene protein. The results of the present study indicated that QC may be a potential anti-DLBCL drug.
\end{abstract}

\section{Introduction}

Diffuse large B-cell lymphoma (DLBCL) is the most common type of lymphoma, representing $25 \%$ of all lymphoproliferative disorders (1). Despite its aggressive disease course, $\sim 50-70 \%$ of patients may experience benefits with R-CHOP (rituximab plus cyclophosphamide, doxorubicin, vincristine and prednisone) chemotherapy (1). However,

Correspondence to: Dr Guifang Ouyang or Dr Qitian Mu, Department of Hematology, Ningbo First Hospital, 59 Liuting Street, Ningbo, Zhejiang 315010, P.R. China

E-mail: ougf@163.com

E-mail: muqitian@163.com

Key words: diffuse large B-cell lymphoma, quinacrine, RNA-binding protein Musashi homolog 2-protein numb homolog signaling pathway, apoptosis, cell cycle there remain certain patients with relapse or disease which is refractory to R-CHOP, ultimately with only $\sim 10 \%$ achieving a cure, requiring aggressive salvage chemotherapy and transplantation (2). Therefore, novel therapeutic drugs are being developed to improve the outcomes of this disease.

Quinacrine [QC; 6-chloro-9-(diethylamino-1-methylbuty pamino)-2-methoxyacridine] is a 9-aminoacridine derivative clinically used as an antimalarial drug, which has additionally been observed to have anti-cancer activity $(3,4)$. A number of studies have suggested that the anti-cancer activity of QC is not associated with its DNA-binding ability, and is mediated via the suppression of survival signaling in cancer cells (3). Simultaneous activation of cellular tumor antigen p53 (p53) and suppression of the phosphatidylinositol-3 kinase/RAC- $\alpha$ serine/threonine-protein kinase/protein kinase mTOR and nuclear factor $(\mathrm{NF})-\kappa \mathrm{B}$ pathways serve an important role in the anti-cancer activity of QC $(3,5,6)$. Additionally, in human colon carcinoma cell lines, QC has been demonstrated to promote tumor necrosis factor ligand superfamily member 10 , oxaliplatin and 5 -fluorouracil cytotoxicity by inducing $\mathrm{NF}-\kappa \mathrm{B}$ inactivation $(6,7)$, QC is a chemosensitizer which is able to enhance chemotherapeutic drug-induced apoptosis in cancer cells (8-11). However, the effect of QC on DLBCL cells has not been reported.

The present study investigated the effects of QC on proliferation and apoptosis in DLBCL cell lines and clarified the possible target molecules of QC in DLBCL cells in vitro.

\section{Materials and methods}

Reagents. QC was obtained from Sigma-Aldrich (Merck KGaA, Darmstadt, Germany; cat. no. Q3251) and dissolved in PBS as a $10 \mathrm{mM}$ stock solution. Dilutions to the required concentrations were made in RPMI-1640 medium. Rabbit polyclonal to RNA-binding protein Musashi homolog 2 (MSI2; cat. no. ab50829) antibody was purchased from Abcam (Cambridge, UK); rabbit monoclonal protein numb homolog (Numb; cat. no. 2761S), Myc proto-oncogene protein (c-Myc; cat. no. 5605), $\beta$-actin (cat. no. 8457S) antibody, rabbit polyclonal poly-ADP ribose polymerase 1 (PARP) antibody (cat. no. 9542S), rabbit monoclonal cyclin-dependent kinase (CDK)6 (cat. no. D4S8S), rabbit monoclonal CDK4 (cat. no. D9G3E) and rabbit polyclonal 
caspase-3 antibody (cat. no. 9665S) were purchased from Cell Signaling Technology, Inc. (Danvers, MA, USA).

Cell culture. DLBCL cell lines OCI-Ly01 and SU-DHL-8 were purchased from the American Type Culture Collection (Manassas, VA, USA) and passaged for $<6$ months following receipt or resuscitation from stocks, and were maintained in RPMI-1640 medium (Hyclone; GE Healthcare Life Sciences, Logan, UT, USA) supplemented with $10 \%$ fetal bovine serum (AusGeneX, Molendinar, Queensland, Australia), 4 mM L-glutamine (Sigma-Aldrich; Merck KGaA), 100 U/ml penicillin (Hyclone; GE Healthcare Life Sciences) and $100 \mathrm{U} / \mathrm{ml}$ streptomycin (Hyclone; GE Healthcare Life Sciences). All cell cultures were performed at $37^{\circ} \mathrm{C}$ in a humidified atmosphere with $5 \% \mathrm{CO}_{2}$.

Cell viability analysis. The cell viability of DLBCL cell lines was measured using the MTS method (CellTiter $96{ }^{\circledR}$ Aqueous One Solution; cat. no. 207284; Promega Corporation, Madison, WI, USA). A total of $2 \times 10^{4}$ cells/well were incubated in quadruplicate in a 96 -well microculture plate, in the presence of different concentrations of QC in a final volume of $0.1 \mathrm{ml}$ for $48 \mathrm{~h}$ at $37^{\circ} \mathrm{C}$. Subsequently, each well was treated with MTS $(20 \mu \mathrm{l} \mathrm{MTS} / 100 \mu \mathrm{l})$ for $4 \mathrm{~h}$, and the absorption values at $590 \mathrm{~nm}$ were determined using an automatic ELISA plate reader (iMark; Bio-Rad Laboratories, Inc., Hercules, CA, USA). Values were normalized to untreated (control) samples.

Cell cycle analysis. Cells $\left(1.0 \times 10^{5} / \mathrm{ml}\right)$ were treated with 0 , $1,1.5$ and $2 \mu \mathrm{mol} / 1 \mathrm{QC}$ for $48 \mathrm{~h}$, and subsequently fixed with $100 \%$ cold ethanol at $-20^{\circ} \mathrm{C}$ for $48 \mathrm{~h}$, followed by staining with a Cell Cycle Staining kit [propidium iodide (PI); MultiSciences Biotech Co., Ltd., Hangzhou, China; cat. no. CCS012] in the presence of RNase for $15 \mathrm{~min}$ at room temperature. Cell-cycle distribution was assessed using a FACScan instrument (BD FACSCanto ${ }^{\mathrm{TM}}$ II; BD Biosciences, Franklin Lakes, NJ, USA). Data were analyzed using FlowJo 7.6.1 software (FlowJo LLC, Ashland, OR, USA).

Analysis of apoptosis. Cells $\left(1.0 \times 10^{5} / \mathrm{ml}\right)$ were treated with $0,0.8,1.6$ and $3.2 \mu \mathrm{mol} / \mathrm{l} \mathrm{QC}$ for $24 \mathrm{~h}$. Staining was performed using annexin $\mathrm{V}$-fluorescein isothiocyanate (Multisciences Biotech Co., Ltd.; cat. no. 4100546) in conjunction with PI, according to the manufacturer's protocol, and was assessed using a FACScan instrument (BD FACSCanto $^{\text {TM }}$ II; BD Biosciences). Data were analyzed using BD FACSDiva software version 3.3.11. Apoptosis was validated via PARP cleavage and analyzed through western blotting.

Protein extraction and western blot analysis. Cells were lysed using SDS buffer (BBI Solutions, Cardiff, UK) containing proteinase inhibitors (phenylmethylsulfonyl fluoride). Cell extracts containing $50 \mu \mathrm{g}$ of proteins, determined by the bicinchoninic acid method, were separated by SDS-PAGE on a $12 \%$ gel, and transferred onto polyvinylidene difluoride membranes (Bio-Rad Laboratories, Inc.). The membrane was blocked in 5\% nonfat milk (Shanghai Bright Diary Group Co., Ltd, Shanghai, China) at room temperature for $2 \mathrm{~h}$ and incubated with specific antibodies $(1: 1,000)$ overnight at $4^{\circ} \mathrm{C}$. Primary antibodies were detected by incubating the membrane in anti-rabbit IgG, HRP-linked antibody (cat. no. 7074; Cell Signaling Technology, Inc.) for $2 \mathrm{~h}$ at room temperature, using enhanced chemiluminescence (PerkinElmer, Inc., Waltham, MA, USA). Densitometry quantification of immunoblot analyses was performed using Image Lab software (version 5.2.1; Bio-Rad Laboratories, Inc.).

Statistical analysis. All statistical analyses were performed using SPSS 17.0 (SPSS, Inc., Chicago, IL, USA). Data are presented as the mean \pm standard deviation. The statistical significance of the differences observed between experimental groups was determined using one-way analysis of variance and a post hoc LSD test. $\mathrm{P}<0.05$ was considered to indicate a statistically significant difference.

\section{Results}

QC inhibits the growth of SU-DHL-8 and OCI-Ly01 cells. The present study investigated whether QC leads to the inhibition of DLBCL cell growth. The two DLBCL cell lines (OCI-Ly01 and SU-DHL-8) were cultured with varying concentrations of QC $(0,2.5,5$ and $10 \mu \mathrm{M})$ for $24 \mathrm{~h}$, and it was observed that the cells exhibited green fluorescence, and that the fluorescence intensity gradually weakened with the increase in QC concentration (Fig. 1A). Cell viability was assessed by MTS assay. As the dose of QC increased from 1 to $8 \mu \mathrm{M}$, cell growth inhibition increased in a dose-dependent manner in the two DLBCL cell lines (Fig. 1B and C). The half-maximal inhibitory concentrations $\left(\mathrm{IC}_{50}\right)$ for SU-DHL-8 and OCI-Ly01 were 2 and $1.8 \mu \mathrm{M}$, respectively. The two cell lines were treated with a variety of different concentrations of QC for $96 \mathrm{~h}$, and cell growth was inhibited in a dose- and time-dependent manner (Fig. 1D and E).

QC arrests the cell cycle of SU-DHL-8 and OCI-LyOI cells at the G0/G1 phase. In order to understand whether the growth inhibitory effect of QC contributed to cell cycle arrest, the effects of QC on the cell cycle were evaluated. It was observed that QC $(2 \mu \mathrm{M})$ induced apparent $\mathrm{G} 0 / \mathrm{G} 1$ phase arrest in OCI-Ly01 compared with control cells $(\mathrm{P}=0.00022$; Fig. 2A and B). QC (1, 1.5 and $2 \mu \mathrm{M})$ was able to decrease the protein expression of CDK4 compared with control cells $(\mathrm{P}=0.002, \mathrm{P}<0.001, \mathrm{P}<0.001)$, and consistent results were observed with CDK6 $(\mathrm{P}<0.001)$ in OCI-Ly01 cells (Fig. 2C and D).

Consistent results were also observed in SU-DHL-8 cells. QC (1.5 and $2 \mu \mathrm{M})$ induced apparent G0/G1 phase arrest compared with the control cells $(\mathrm{P}=0.002, \mathrm{P}=0.0002)$ (Fig. 3A and B), the protein expression of CDK4 decreased following treatment with $\mathrm{QC}(1,1.5$ and $2 \mu \mathrm{M})$ compared with control cells $(\mathrm{P}<0.001$, respectively) and the same was observed for CDK6 ( $\mathrm{P}<0.001)$ (Fig. 3C and 3D).

QC induces apoptosis of SU-DHL-8 and OCI-LyO1 cell lines. In order to study the induction of apoptosis, SU-DHL-8 and OCI-Ly01 cells were treated with four different QC concentrations, $0,0.8,1.6$ and $3.2 \mu \mathrm{M}$, for $24 \mathrm{~h}$. Compared 
A

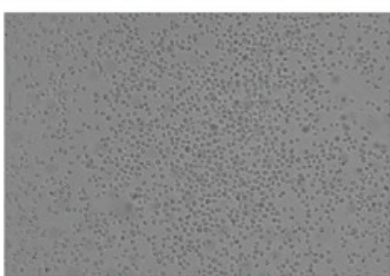

Control

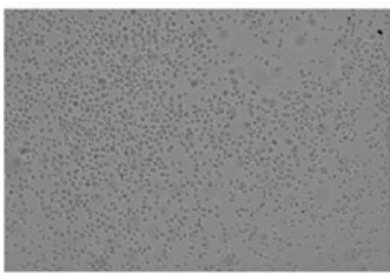

Control

B

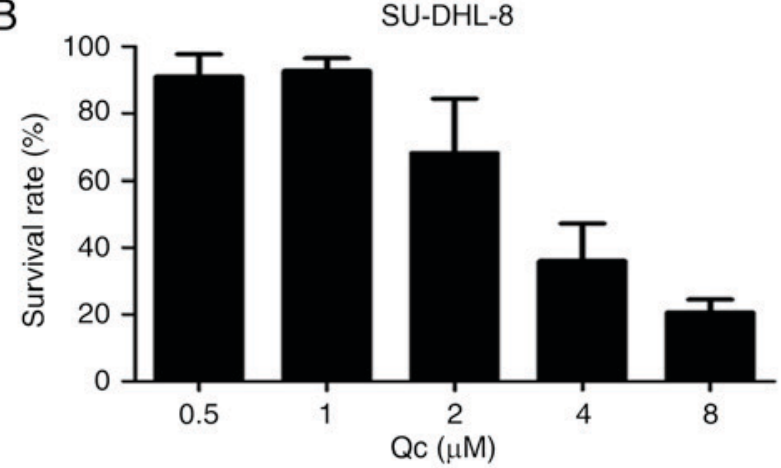

D

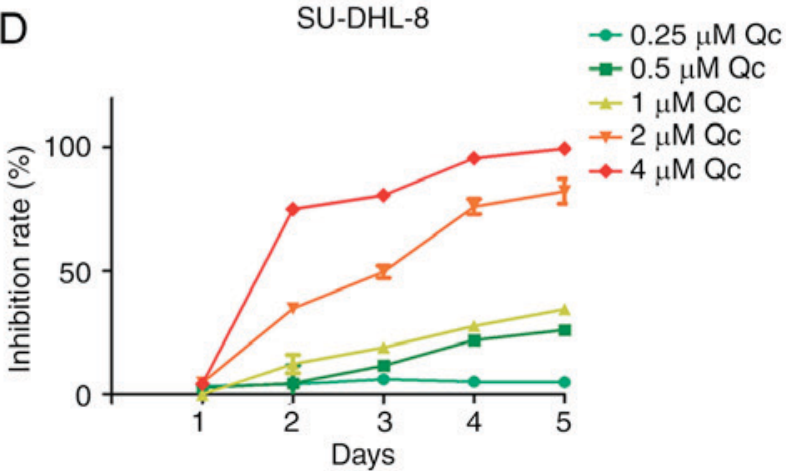

SU-DHL-8

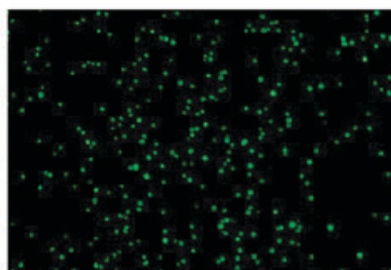

$2.5 \mu \mathrm{M} Q \mathrm{c}$

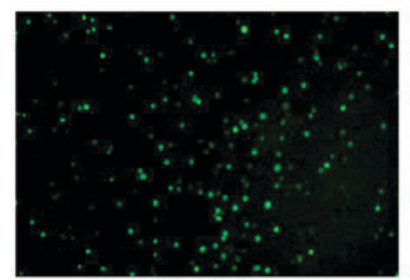

$5 \mu \mathrm{M} Q \mathrm{c}$

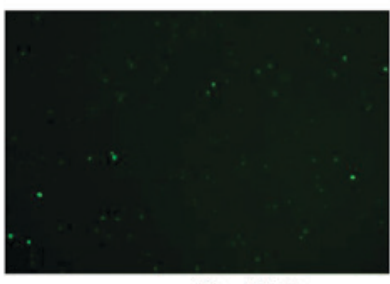

$10 \mu \mathrm{M} Q \mathrm{c}$
OCl-Ly01

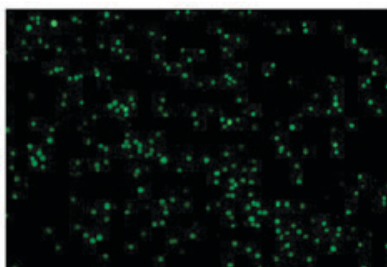

$2.5 \mu \mathrm{M}$ Qc

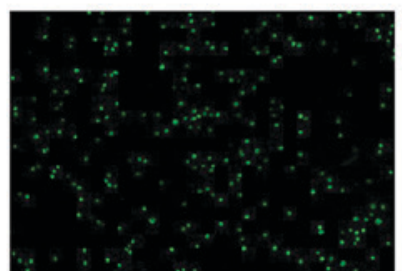

$5 \mu \mathrm{M}$ Qc

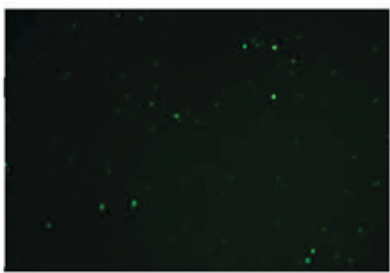

$10 \mu \mathrm{M}$ Qc
C

OCl-Ly01

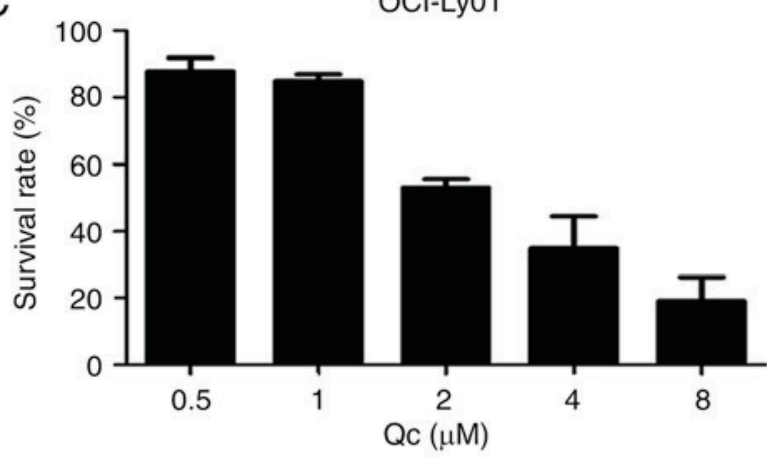

E

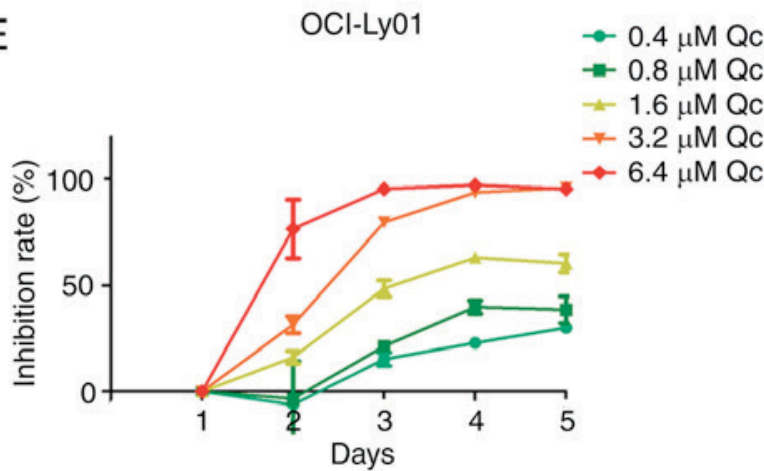

Figure 1. QC inhibits the proliferation of diffuse large B-cell lymphoma cells. (A) DLBCL cell lines (SU-DHL-8 and Ly01) were incubated with QC and exhibited green fluorescence. Fluorescence intensity gradually weakened with the increase in drug concentration o. Magnification, x100. (B) Viability of SU-DHL-8 cells and (C) OCI-Ly01 cells following treatment with QC for $48 \mathrm{~h}$. The effect of QC on the growth of the (D) SU-DHL-8 and (E) OCI-Ly01 cell lines is presented. QC, quinacrine.

with the control group, the percentages of apoptotic cells of groups treated with QC increased significantly in a dose-dependent manner in SU-DHL-8 and OCI-Ly01 cells (Fig. 4A and B). In addition, the expression levels of cleaved PARP protein increased in groups treated with QC compared with control cells in the OCI-Ly01 cell line $(\mathrm{P}<0.001)$, and the same results were noted in the SU-DHL- 8 cell line $(\mathrm{P}<0.001, \mathrm{P}=0.007$ and $\mathrm{P}<0.001$, respectively) (Fig. 4C and $\mathrm{D})$.

$Q C$ regulates the MSI2-NUMB-c-Myc signaling pathway. It was previously reported that MSI2 served an important role in hematopoietic stem cell function and cell fate determination (12). MSI2 inhibits the expression of Numb, which is an evolutionarily well-conserved protein $(13,14)$. Therefore, the present study examined whether QC may affect the MSI2-Numb signaling pathway. SU-DHL-8 and OCI-Ly01 constitutively expressed MSI2 and Numb. When SU-DHL-8 and OCI-Ly01 were treated with $0,1.6,3.2$ and $6.4 \mu \mathrm{M}$ QC for $24 \mathrm{~h}$, the expression of MSI2 decreased, whereas that of Numb increased (Fig. 5A and B), suggesting that the inhibition of MSI2 may lead to the accumulation of Numb protein. Previous studies have demonstrated that c-Myc 
A

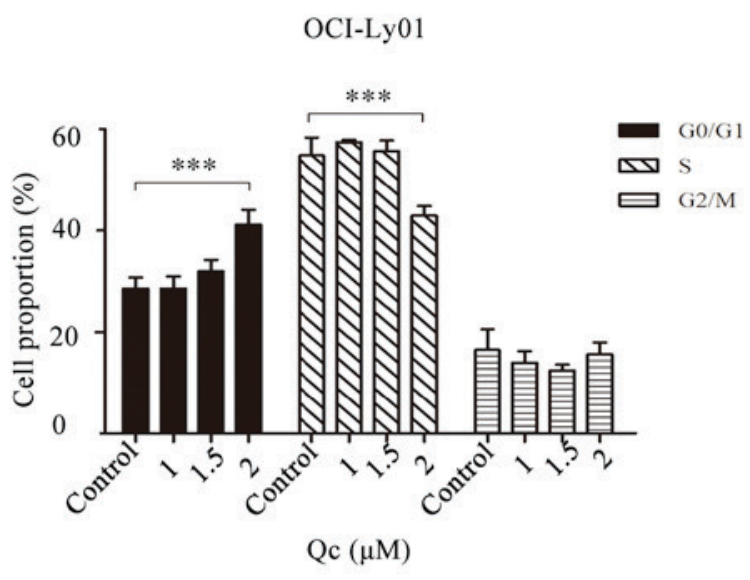

C

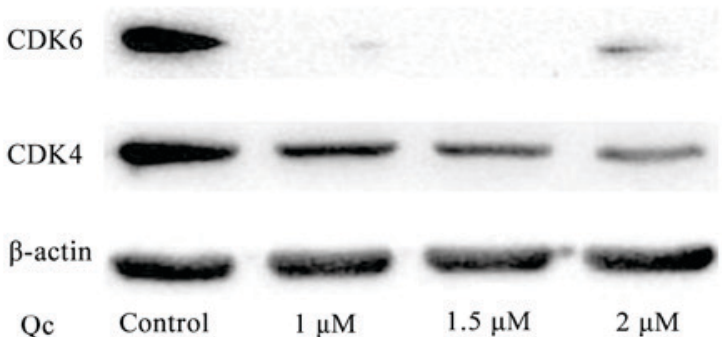

B
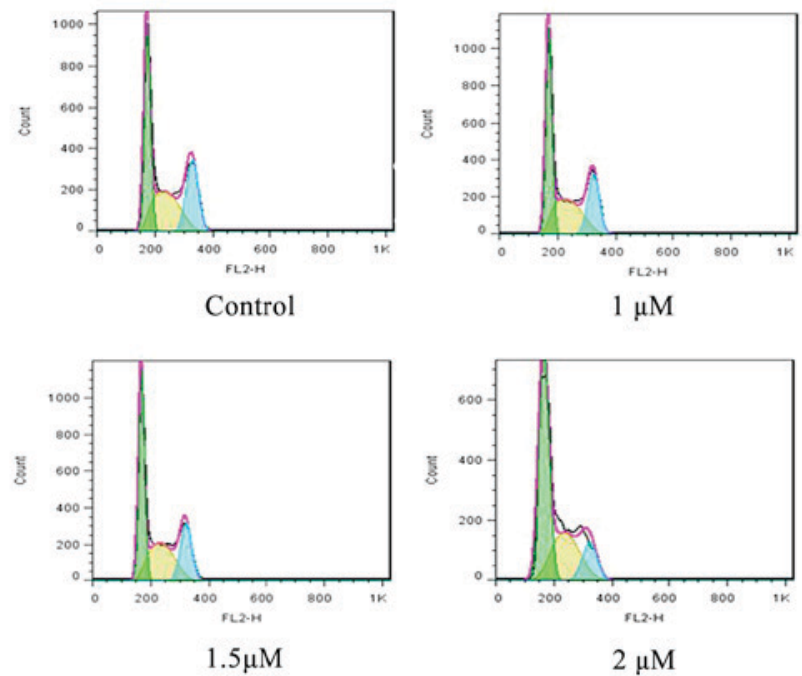

D

OCI-Ly01

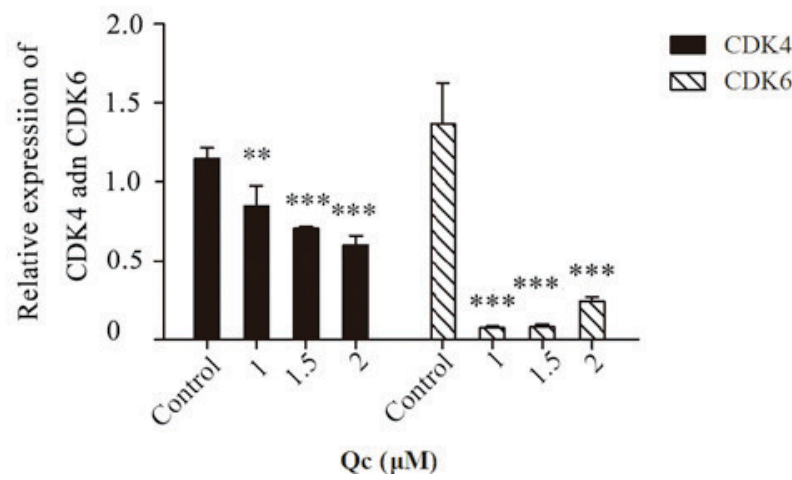

Figure 2. QC induces cell cycle arrest of OCI-Ly01 cells at the G0/G1 phase. (A) Analysis of the (B) flow cytometry data demonstrated cell cycle arrest at the G0/G1 phase. The protein expression of CDK4/CDK6 decreased in OCI-Ly01 cells following treatment, as demonstrated by (C) western blotting and (D) densitometric analysis. Each experiment was performed in triplicate, and error bars represent the mean \pm standard deviation. ${ }^{* *} \mathrm{P}<0.01 ;{ }^{* * *} \mathrm{P}<0.001 \mathrm{vs}$. control. QC, quinacrine; CDK, cyclin-dependent kinase.

was regulated by Numb (15). Therefore, the present study assessed the expression level of c-Myc by western blotting. As hypothesized, QC decreased c-Myc expression with the increase of drug concentration in SU-DHL-8 and OCI-Ly01 cells (Fig. 5C and D).

\section{Discussion}

QC is a well-known antimalarial drug, and its anticancer effects have been demonstrated (16-19). It has been demonstrated that QC may induce apoptosis and arrest the cell cycle at the $\mathrm{S}$ phase via inhibition of topoisomerase activity, and induction of p53 and CDK inhibitor 1 in breast cancer and colon cancer cells $(4,20)$, and inhibition of $N F-\kappa B$ and Wnt-T-cell factor signaling via the adenomatous polyposis coli gene in breast cancer (21). However, to the best of our knowledge, the inhibition of QC in DLBCL cells has been not reported. The present study investigated the antiproliferative potential of QC in SU-DHL-8 and OCI-Ly01 cells. It was observed that QC inhibited cell growth. The $\mathrm{IC}_{50}$ of QC in SU-DHL-8 and OCI-Ly01 cells was 1.8 and $2 \mu \mathrm{M}$, respectively, approximately equal to the $\mathrm{IC}_{50}$ of $\mathrm{QC}$ in other cancer cells, including non-small cell lung cancer (22), breast cancer (4) and leukemia K562 cells (23). The increased protein expression of cleaved PARP following treatment with QC indicated that QC was able to induce cellular apoptosis in OCI-Ly01 and SU-DHL-8 cell lines, which was consistent with previous reports $(4,5,11)$. QC additionally induced cell cycle arrest at the G0/G1 phase and apoptosis in human DLBCL cell lines SU-DHL-8 and OCI-Ly01 in a dose-dependent manner, and the decreased expression of CDK6 and CDK4 confirmed G0/G1 cell cycle arrest. However, previous reports indicated that QC induced cell cycle arrest at the G1/S and G2/M (22), and sub-G1 and $\mathrm{S}$ phases (24), which is not consistent with the results of the present study. A possible reason for this is that the 
A

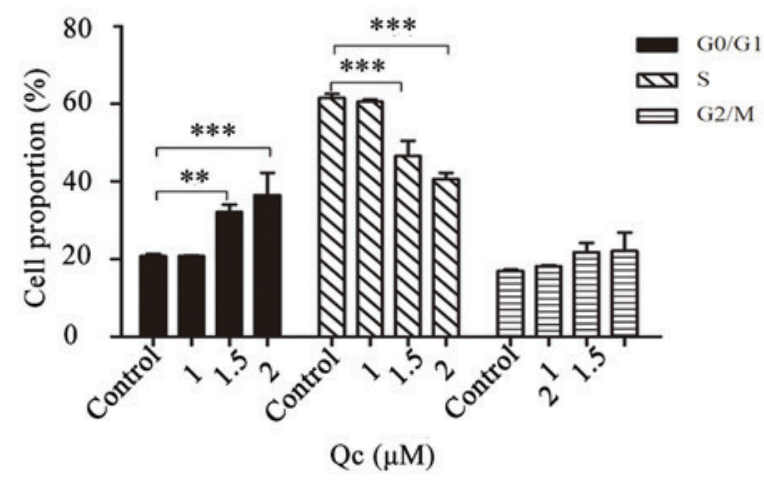

C

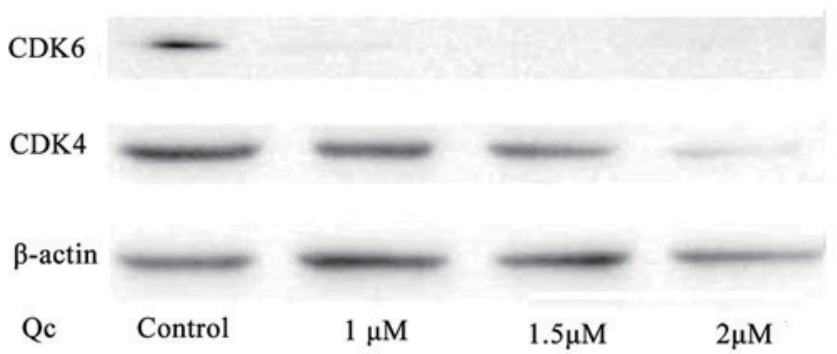

B

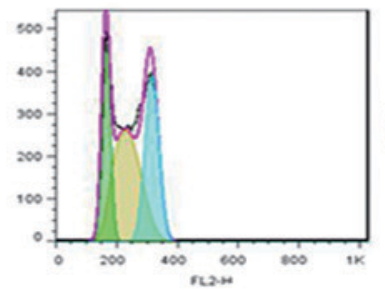

Control

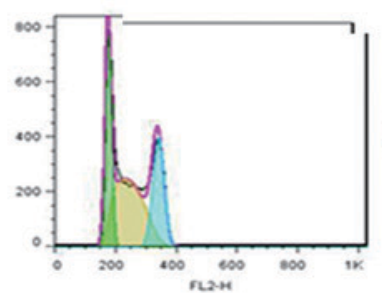

$1.5 \mu \mathrm{M}$

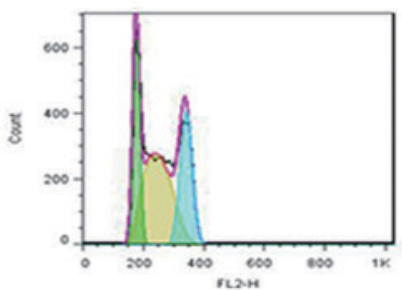

$1 \mu \mathrm{M}$

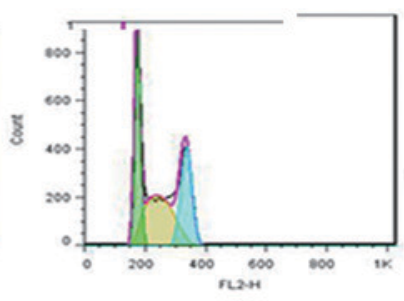

$2 \mu \mathrm{M}$
D

SU-DHL-8

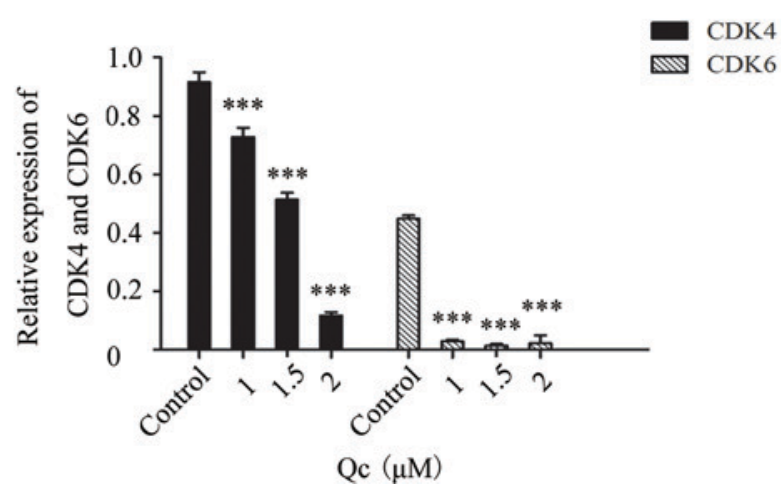

Figure 3. QC induces cell cycle arrest of SU-DHL-8 cells in G0/G1 phase. (A) Analysis of the (B) flow cytometry data demonstrated cell cycle arrest at the G0/G1 phase. The protein expression of CDK4/CDK6 decreased in SU-DHL-8 cells following treatment, as demonstrated by (C) western blotting and (D) densitometric analysis. Each experiment was performed in triplicate, and error bars represent the mean \pm standard deviation. ${ }^{* *} \mathrm{P}<0.01 ;{ }^{* * * *} \mathrm{P}<0.001 \mathrm{vs}$. control. QC, quinacrine; CDK, cyclin-dependent kinase.

molecular mechanism of QC is heterogeneous in different types of cells.

The Musashi proteins are RNA-binding proteins which are encoded by two translational regulatory genes, MSI1 and MSI2, located on chromosomes 12 and $17(25,26)$. They distinctively regulate transcriptional events and act as cell cycle regulators (27). The Musashi gene family is highly expressed in stem cells $(28,29)$. MSI1 and MSI2 expression has been associated with an unfavorable prognosis in several types of tumor, including glioma, pediatric brain tumor, breast cancer and colorectal cancer $(30,31)$. In previous studies, high MSI2 expression indicated a poor prognosis and facilitated risk and treatment stratification in adult and pediatric patients with B-cell acute lymphoblastic leukemia $(32,33)$, indicating that MSI2 was able to serve an important role in B-cell neoplasm. In the present study, MSI2 expression was detected in SU-DHL-8 and OCI-Ly01 cells, which further supported this hypothesis. Notably, the results of the present study demonstrated that QC was able to downregulate MSI2 expression and upregulate Numb expression. Previous studies demonstrated that MSI2 was able to inhibit Numb mRNA translation, promoting the development and progression of pancreatic cancer (34).

In addition, the knockdown of Numb has been demonstrated to increase c-Myc expression (15). c-Myc is the master transcription factor of cell proliferation and is involved in numerous hematological and solid types of cancer (35), including DLBCL (36). In the present study, it was observed that QC induced cell cycle arrest at the G0/G1 phase. Consistent with the results of the present study, downregulation of MSI2 and c-Myc has been observed to induce cell cycle arrest at the G0/G1 phase (37) and Numb overexpression may inhibit cell cycle progression at the G0/G1 phase $(38,39)$. Additionally, CDK4 and CDK6 are two classical cell cycle-associated proteins which are involved in cell cycle transformation between the G0/G1 and S phases. It 
A

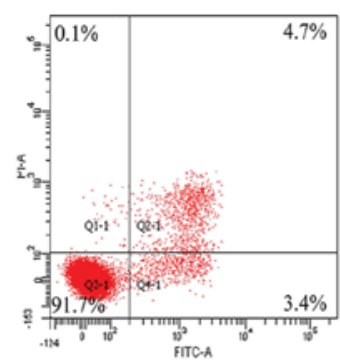

Control

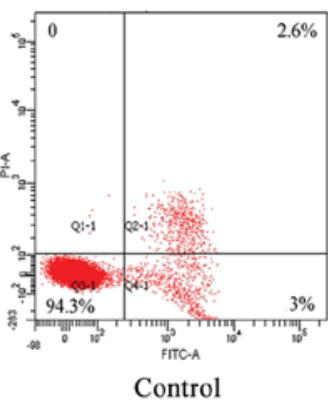

Control
SU-DHL-8

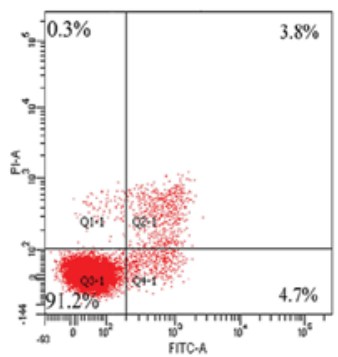

$0.8 \mu \mathrm{M} Q \mathrm{c}$

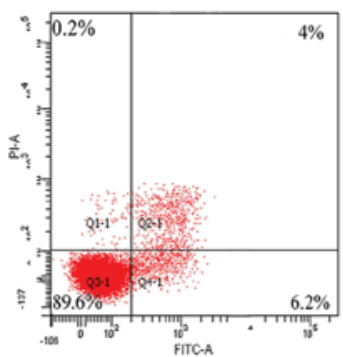

$1.6 \mu \mathrm{M}$ Qc

OCI-LY01

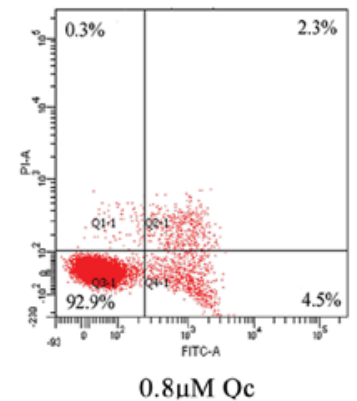

SU-DHL-8

$\triangle$ OCI-Ly01

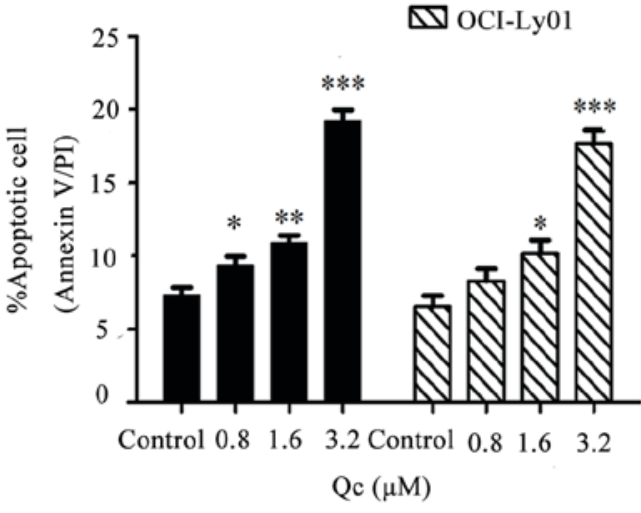

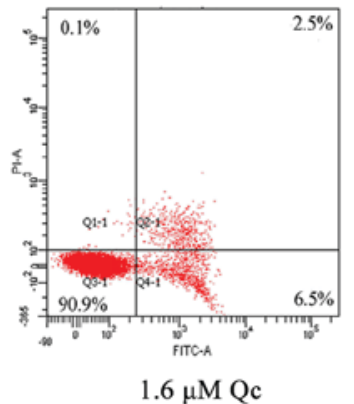

$1.6 \mu \mathrm{M} \mathrm{Qc}$
B

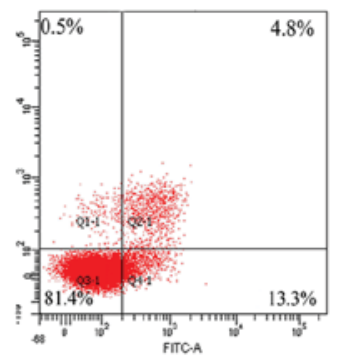

$3.2 \mu \mathrm{M} Q \mathrm{c}$

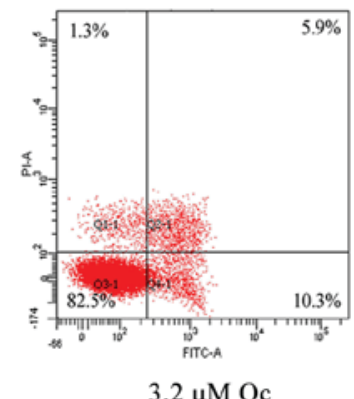

$3.2 \mu \mathrm{M}$ Qc

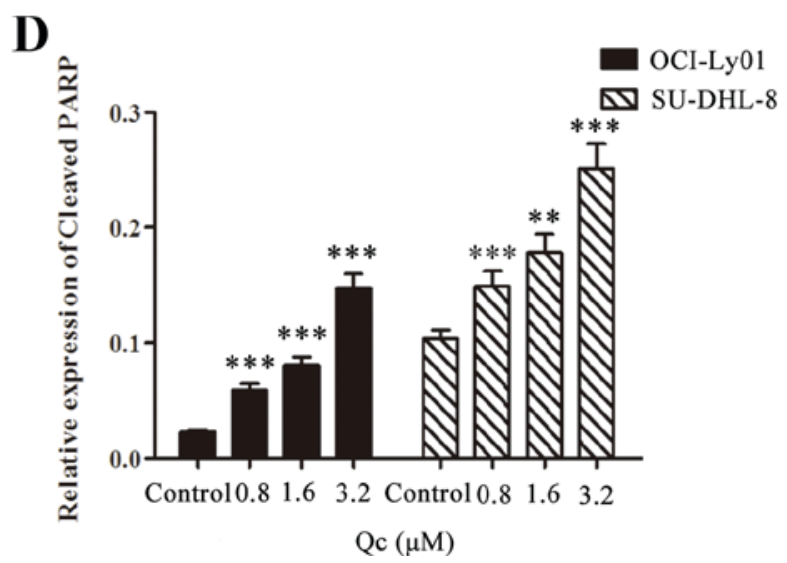

Figure 4. QC induces apoptosis in vitro in diffuse large B-cell lymphoma cells. SU-DHL-8 and OCI-Ly01 cells were treated with QC for 24 h, and subjected to (A) flow cytometry and (B) statistical analysis. Cl-PARP expression following treatment with QC for $24 \mathrm{~h}$ in SU-DHL-8 and OCI-Ly01 cells was examined via (C) western blotting and (D) densitometric analysis. Each experiment was performed in triplicate, and error bars represent the mean \pm standard deviation. ${ }^{*} \mathrm{P}<0.05 ;{ }^{* *} \mathrm{P}<0.01 ;{ }^{* * *} \mathrm{P}<0.001$ vs. control. QC, quinacrine; PARP, poly-ADP ribose polymerase 1; Fl, full-length; $\mathrm{Cl}$, cleaved.

was observed that QC decreased CDK4 and CDK6 expression in the OCI-Ly01 and SU-DHL-8 cell lines, consistent with the observed G0/G1 cell cycle arrest. Therefore, it was hypothesized that QC may inhibit MSI2 expression, increase 
A

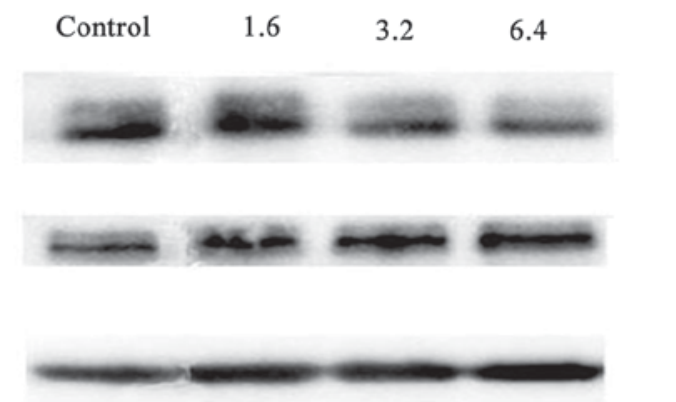

Qc $(\mu \mathrm{M}) \quad$ Control

1.6

3.2

6.4

MSI2

NUMB
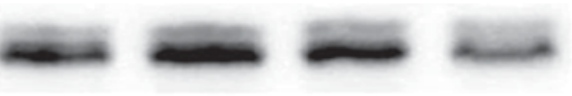

$\beta$-actin

OCI-Ly01

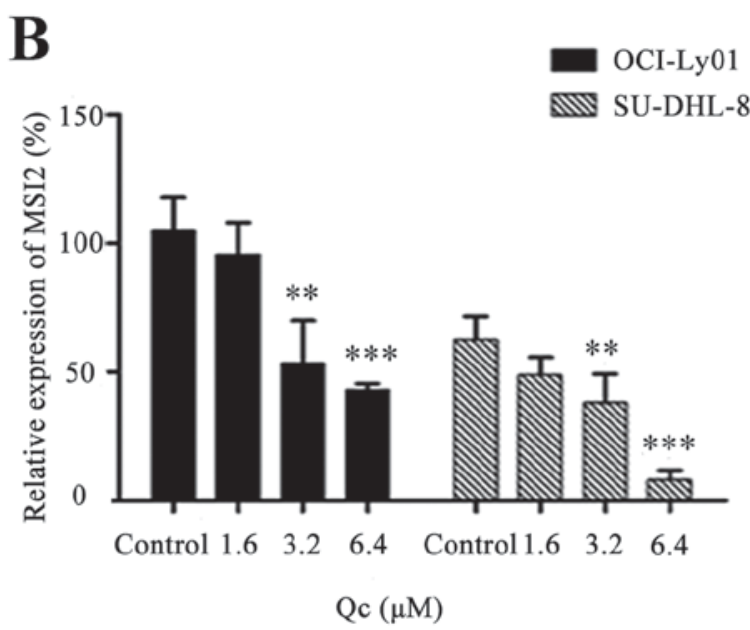

C

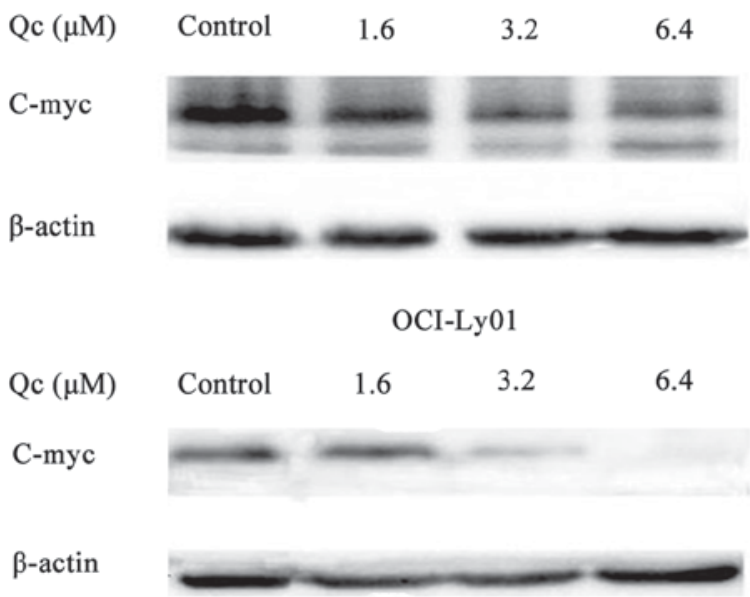

SU-DHL-8

D

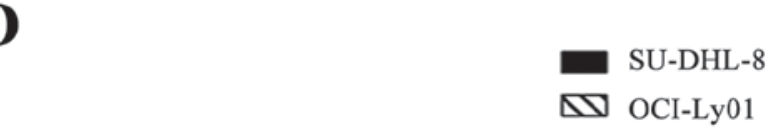

Figure 5. QC regulates the expression of MSI2, Numb and c-Myc proteins in SU-DHL-8 and OCI-Ly01 cells. The expression of MSI2 declined and the expression of Numb increased, as demonstrated by (A) western blotting and (B) densitometric analysis. The expression of c-Myc decreased following treatment with QC, as demonstrated by (C) western blotting and (D) densitometric analysis. $\beta$-actin was used as the loading control. Each experiment was performed in triplicate, and error bars represent the mean \pm standard deviation. ${ }^{*} \mathrm{P}<0.05 ;{ }^{* * *} \mathrm{P}<0.01 ;{ }^{* * * *} \mathrm{P}<0.001$ vs. control. QC, quinacrine; MSI2, RNA-binding protein Musashi homolog 2; Numb, protein numb homolog; c-Myc, Myc proto-oncogene protein.

NUMB expression, suppress c-Myc expression, and decrease CDK4 and CDK6 expression.

In conclusion, the results of the present study indicated that QC may inhibit the growth of DLBCL cells, possibly via the MSI2-NUMB signaling pathway, and is a potential drug for the treatment of DLBCL. However, further studies in vivo are required to confirm the clinical effects of QC in DLBCL. 


\section{Acknowledgements}

The present study was supported by the Natural Science Foundation of Zhejiang Province (grant nos. LY17H160005 and LY14H080001), the Project from Traditional Chinese Medicine Administration of Zhejiang Province (grant no. 2015ZZ018) and the Natural Science Foundation of Ningbo (grant no. 2014A610217).

\section{References}

1. Coiffier B, Thieblemont C, Van Den Neste E, Lepeu G, Plantier I, Castaigne S, Lefort S, Marit G, Macro M, Sebban C, et al: Long-term outcome of patients in the LNH-98.5 trial, the first randomized study comparing rituximab-CHOP to standard CHOP chemotherapy in DLBCL patients: A study by the Groupe d'Etudes des Lymphomes de l'Adulte. Blood 116: 2040-2045, 2010.

2. Friedberg JW: Relapsed/refractory diffuse large B-cell lymphoma. Hematology Am Soc Hematol Educ Program 2011: 498-505, 2011.

3. Ehsanian R, Van Waes C and Feller SM: Beyond DNA binding-a review of the potential mechanisms mediating quinacrine's therapeutic activities in parasitic infections, inflammation, and cancers. Cell Commun Signal 9: 13, 2011.

4. Preet R, Mohapatra P, Mohanty S, Sahu SK, Choudhuri T, Wyatt MD and Kundu CN: Quinacrine has anticancer activity in breast cancer cells through inhibition of topoisomerase activity. Int J Cancer 130: 1660-1670, 2012.

5. Wang W, Ho WC, Dicker DT, MacKinnon C, Winkler JD, Marmorstein R and El-Deiry WS: Acridine derivatives activate p53 and induce tumor cell death through Bax. Cancer Biol Ther 4: 893-898, 2005.

6. Gallant JN, Allen JE, Smith CD, Dicker DT, Wang W, Dolloff NG, Navaraj A and El-Deiry WS: Quinacrine synergizes with 5-fluorouracil and other therapies in colorectal cancer. Cancer Bio Ther 12: 239-251, 2011.

7. Jani TS, DeVecchio J, Mazumdar T, Agyeman A and Houghton JA: Inhibition of NF-kappaB signaling by quinacrine is cytotoxic to human colon carcinoma cell lines and is synergistic in combination with tumor necrosis factor-related apoptosis-inducing ligand (TRAIL) or oxaliplatin. J Biol Chem 285: 19162-19172, 2010.

8. Friedman J, Nottingham L, Duggal P, Pernas FG, Yan B, Yang XP, Chen Z and Van Waes C: Deficient TP53 expression, function, and cisplatin sensitivity are restored by quinacrine in head and neck cancer. Clin Cancer Res 13: 6568-6578, 2007.

9. Wang Y, Bi Q, Dong L, Li X, Ge X, Zhang X, Fu J, Wu D and Li S: Quinacrine enhances cisplatin-induced cytotoxicity in four cancer cell lines. Chemotherapy 56: 127-134, 2010.

10. Wang W, Gallant JN, Katz SI, Dolloff NG, Smith CD, Abdulghani J, Allen JE, Dicker DT, Hong B, Navaraj A and El-Deiry WS: Quinacrine sensitizes hepatocellular carcinoma cells to TRAIL and chemotherapeutic agents. Cancer Biol Ther 12: 229-238, 2011

11. Wu X, Wang Y, Wang H, Wang Q, Wang L, Miao J, Cui F and Wang J: Quinacrine inhibits cell growth and induces apoptosis in human gastric cancer cell line SGC-7901. Curr Ther Res Clin Exp 73: 52-64, 2012.

12. Park SM, Deering RP, Lu Y, Tivnan P, Lianoglou S, Al-Shahrour F, Ebert BL, Hacohen N, Leslie C, Daley GQ, et al: Musashi-2 controls cell fate, lineage bias, and TGF- $\beta$ signaling in HSCs. J Exp Med 211: 71-87, 2014.

13. Zhong W, Feder JN, Jiang MM, Jan LY and Jan YN: Asymmetric localization of a mammalian numb homolog during mouse cortical neurogenesis. Neuron 17: 43-53, 1996.

14. Guo M, Jan LY and Jan YN: Control of daughter cell fates during asymmetric division: Interaction of Numb and Notch. Neuron 17: 27-41, 1996.

15. Lu Y, Xu W, Ji J, Feng D, Sourbier C, Yang Y, Qu J, Zeng Z, Wang C, Chang X, et al: Alternative splicing of the cell fate determinant Numb in hepatocellular carcinoma. Hepatology 62: 1122-1131, 2015.

16. Gurova K: New hopes from old drugs: Revisiting DNA-binding small molecules as anticancer agents. Future Oncol 5: 1685-1704, 2009.
17. Neznanov N, Gorbachev AV, Neznanova L, Komarov AP, Gurova KV, Gasparian AV, Banerjee AK, Almasan A, Fairchild RL and Gudkov AV: Anti-malaria drug blocks proteotoxic stress response: Anti-cancer implications. Cell Cycle 8: 3960-3970, 2009.

18. Guo C, Gasparian AV, Zhuang Z, Bosykh DA, Komar AA, Gudkov AV and Gurova KV: 9-Aminoacridine-based anticancer drugs target the PI3K/AKT/mTOR, NF-kappaB and p53 pathways. Oncogene 28: 1151-1161, 2009.

19. Gurova KV, Hill JE, Guo C, Prokvolit A, Burdelya LG, Samoylova E, Khodyakova AV, Ganapathi R, Ganapathi M, Tararova ND, et al: Small molecules that reactivate p53 in renal cell carcinoma reveal a NF-kappaB-dependent mechanism of p53 suppression in tumors. Proc Natl Acad Sci USA 102: 17448-17453, 2005.

20. Mohapatra P, Preet R, Das D, Satapathy SR, Choudhuri T, Wyatt MD and Kundu CN: Quinacrine-mediated autophagy and apoptosis in colon cancer cells is through a p53- and p21-dependent mechanism. Oncol Res 20: 81-91, 2012.

21. Preet R, Mohapatra P, Das D, Satapathy SR, Choudhuri T, Wyatt MD and Kundu CN: Lycopene synergistically enhances quinacrine action to inhibit Wnt-TCF signaling in breast cancer cells through APC. Carcinogenesis 34: 277-286, 2013

22. Dermawan JK, Gurova K, Pink J, Dowlati A, De S, Narla G, Sharma N and Stark GR: Quinacrine overcomes resistance to erlotinib by inhibiting FACT, $\mathrm{NF}-\kappa \mathrm{B}$, and cell-cycle progression in non-small cell lung cancer. Mol Cancer Ther 13: 2203-2214, 2014.

23. Changchien JJ, Chen YJ, Huang CH, Cheng TL, Lin SR and Chang LS: Quinacrine induces apoptosis in human leukemia K562 cells via p38 MAPK-elicited BCL2 down-regulation and suppression of ERK/c-Jun-mediated BCL2L1 expression. Toxicol Appl Pharmacol 284: 33-41, 2015.

24. Preet R, Siddharth S, Satapathy SR, Das S, Nayak A, Das D, Wyatt MD and Kundu CN: Chk1 inhibitor synergizes quinacrine mediated apoptosis in breast cancer cells by compromising the base excision repair cascade. Biochem Pharmacol 105: 23-33, 2016.

25. Barbouti A, Höglund M, Johansson B, Lassen C, Nilsson PG, Hagemeijer A, Mitelman F and Fioretos T: A novel gene, MSI2, encoding a putative RNA-binding protein is recurrently rearranged at disease progression of chronic myeloid leukemia and forms a fusion gene with HOXA9 as a result of the cryptic $t(7 ; 17)$ (p15;q23). Cancer Res 63: 1202-1206, 2003.

26. Kawahara H, Imai T, Imataka H, Tsujimoto M, Matsumoto $\mathrm{K}$ and Okano H: Neural RNA-binding protein Musashil inhibits translation initiation by competing with eIF4G for PABP. J Cell Biol 181: 639-653, 2008.

27. Imai T, Tokunaga A, Yoshida T, Hashimoto M, Mikoshiba K, Weinmaster G, Nakafuku $M$ and Okano $H$ : The neural RNA-binding protein Musashil translationally regulates mammalian numb gene expression by interacting with its mRNA. Mol Cell Biol 21: 3888-3900, 2001

28. Kong DS, Kim MH, Park WY, Suh YL, Lee JI, Park K, Kim JH and Nam DH: The progression of gliomas is associated with cancer stem cell phenotype. Oncol Rep 19: 639-643, 2008.

29. Siddall NA, McLaughlin EA, Marriner NL and Hime GR: The RNA-binding protein Musashi is required intrinsically to maintain stem cell identity. Proc Natl Acad Sci USA 103: 8402-8407, 2006.

30. Wang XY, Yin Y, Yuan H, Sakamaki T, Okano H and Glazer RI: Musashil modulates mammary progenitor cell expansion through proliferin-mediated activation of the Wnt and Notch pathways. Mol Cell Biol 28: 3589-3599, 2008.

31. Sureban SM, May R, George RJ, Dieckgraefe BK, McLeod HL, Ramalingam S, Bishnupuri KS, Natarajan G, Anant S and Houchen CW: Knockdown of RNA binding protein musashi-1 leads to tumor regression in vivo. Gastroenterology 134: 1448-1458, 2008

32. Mu Q, Wang Y, Chen B, Qian W, Meng H, Tong H, Chen F, Ma Q, Ni W, Chen S and Jin J: High expression of Musashi-2 indicates poor prognosis in adult B-cell acute lymphoblastic leukemia. Leuk Res 37: 922-927, 2013.

33. Aly RM and Ghazy HF: Prognostic significance of MSI2 predicts unfavorable outcome in adult B-acute lymphoblastic leukemia. Int J Lab Hematol 37: 272-278, 2015.

34. Sheng W, Dong M, Chen C, Li Y, Liu Q and Dong Q: Musashi2 promotes the development and progression of pancreatic cancer by down-regulating Numb protein. Oncotarget 8: 14359-14373, 2017. 
35. Wierstra I and Alves J: The c-myc promoter: Still MysterY and challenge. Adv Cancer Res 99: 113-333, 2008.

36. Valera A, López-Guillermo A, Cardesa-Salzmann T, Climent F, González-Barca E, Mercadal S, Espinosa I, Novelli S, Briones J, Mate JL, et al: MYC protein expression and genetic alterations have prognostic impact in patients with diffuse large B-cell lymphoma treated with immunochemotherapy. Haematologica 98: 1554-1562, 2013.

37. Han Y, Ye A, Zhang Y, Cai Z, Wang W, Sun L, Jiang S, Wu J, $\mathrm{Yu} \mathrm{K}$ and Zhang S: Musashi-2 silencing exerts potent activity against acute myeloid leukemia and enhances chemosensitivity to daunorubicin. PLoS One 10: $\mathrm{e} 0136484,2015$.
38. Sima J, Zhang B, Yu Y, Sima X and Mao Y: Overexpression of Numb suppresses growth, migration, and invasion of human clear cell renal cell carcinoma cells. Tumour Biol 36: 2885-2892, 2015.

39. Schweinfest CW, Fujiwara S, Lau LF and Papas TS: c-myc can induce expression of $\mathrm{G} 0 / \mathrm{G} 1$ transition genes. Mol Cell Biol 8: 3080-3087, 1988 